\title{
Percutaneous Endoscopic Treatment of Complicated Delayed Bleeding Postpercutaneous Nephrolithotomy: A Novel Suggestion
}

\author{
Akbar Nouralizadeh, MD, Arsalan Aslani, MD, Iman Ghanaat, MD, and Milad Bonakdar Hashemi, MD
}

\begin{abstract}
Background: Delayed bleeding after percutaneous nephrolithotomy (PCNL), which may occur within the first 3 weeks postoperatively, is a life-threatening complication that may result from arteriovenous fistula and arterial pseudoaneurysm. Angioembolization is the standard treatment when these patients develop hemodynamic instability despite conservative measures. Contrast hypersensitivity and renal insufficiency, however, contraindicate angiogram and subsequent embolization; in these patients, alternative methods such as the one described in this study may help in resolving the renal hemorrhage.

Case Presentation: In this case series, we report the effective management of post-PCNL hemorrhage with nephroscopy and nephrostomy and drainage and tamponade because angioembolization was not feasible.

Conclusion: Delayed bleeding after PCNL may be managed conservatively with nephrostomy drainage and tamponade when angioembolization is not feasible.
\end{abstract}

Keywords: percutaneous nephrolithotomy, delayed bleeding, angioembolization failure

\section{Introduction}

$\mathbf{U}$ ROLITHIASIS IS A COMMON disease the prevalence of which is increasing every day.

Percutaneous nephrolithotomy (PCNL) is an effective and common treatment technique for especially large and complex renal calculi. Although PCNL is a common procedure, it can be associated with some mortal or morbid complications such as septicemia, delayed bleeding, and pleural or colonic injury.

Delayed bleeding usually occurs during the first 3 weeks after surgery. ${ }^{1,2}$ Most delayed bleeding can be relieved with conservative management, but $1 \%$ of cases require intervention. ${ }^{1,3}$ Arteriovenous fistula and arterial pseudoaneurysm are the main causes of delayed bleeding. ${ }^{4}$ Angiographic embolization is the standard treatment for patients with delayed bleeding who do not respond to conservative treatment. ${ }^{1,4}$ Angioembolization is not feasible in patients with hypersensitivity to contrast material, patients with normal CT-angiography and patients with low glomerular filtration rates. Angioembolization is not effective in all patients, and sometimes it is accompanied by morbidities and failures that complicate the management of hematuria. Treating recurrent bleeding after angiographic embolization is challenging, and no consensus on the most effective approach was found in the literature. ${ }^{4}$

In our case series, we report the effective management of post-PCNL intractable bleeding with repeat nephroscopy, evacuation of intrarenal blood clots and drainage and tamponade with catheter balloon, because angioembolization was not feasible.

\section{Case Presentation}

\section{Case 1}

A 44-year-old man came to the emergency room with clot passing. The patient had undergone a PCNL 2 weeks before this visit. He was admitted to the hospital, and the necessary orders were implemented. The orders included were close observation, complete bed rest, intravenous hydration, check hemoglobin every 6 hours, prepare adequate blood pack cells, and CT-angiography if feasible. Upon admission, his laboratory data revealed the following levels: hemoglobin $(\mathrm{Hb}): 10.1$, hematocrit: 30.3 , blood urea nitrogen (BUN): 25 , creatinine $(\mathrm{Cr}): 1$. The patient was not responding to conservative management; therefore, a CT-angiography was done. The results were normal with no evidence of visible vascular abnormities. The patient was scheduled for repeat nephroscopy. 


\section{Case 2}

A 38-year-old man was seen at the endourology clinic 7 days post-PCNL with complaints of recurrent passing of many worm-shaped clots. The patient was admitted and the necessary orders were implemented. The orders included were close observation, complete bed rest, intravenous hydration, check hemoglobin every 6 hours, prepare adequate blood pack cells, and CT-angiography if feasible. His laboratory data upon admission revealed the following levels: $\mathrm{Hb}$ : 9.3, hematocrit: $28.1, \mathrm{BUN}$ : $25, \mathrm{Cr}: 1.4$. The patient had a history of hypersensitivity to contrast material during intravenous contrast material injection, so he was scheduled for repeat nephroscopy without any imaging.

\section{Case 3}

A 65-year-old woman came to the emergency room with cola-colored urine. She had a history of chronic kidney disease related to diabetes mellitus and had undergone PCNL 2 weeks before this visit. Her laboratory data upon admission revealed the following levels: $\mathrm{Hb}: 10.6$, Hematocrit: $31.4, \mathrm{Cr}$ : 5, BUN: 45. The radiologist and interventional radiologist refused to use contrast material, so the patient was scheduled for repeat nephroscopy without imaging.

\section{Case 4}

A 55-year-old man came into the emergency room with complaints of dizziness, cold sweats, and tea-colored urine with clot passing. The patient was admitted and the proper orders were implemented. The orders included were close observation, complete bed rest, intravenous hydration, check hemoglobin every 6 hours, prepare adequate blood pack cells, and CT-angiography if feasible. His laboratory data upon admission revealed the following levels: $\mathrm{Hb}: 7.8$, hematocrit: 22.7, BUN: 30, Cr: 1. CT-angiography was performed, and a pseudoaneurysm was seen at the upper pole of the kidney. The patient, with full knowledge of the possible complications, deliberately chose repeat nephroscopy.

\section{Case 5}

A 50-year-old man was referred to our endourologist ward by our colleagues. His chief complaint was worm-shaped clot passing 2 weeks post-PCNL. He underwent CT-angiography that revealed vascular abnormalities at the lower pole of the involved kidney. The patient consciously refused angiographic embolization with knowledge of the possible complications and deliberately chose repeat nephroscopy.

\section{Case 6}

A 63-year-old man was referred at the endourology clinic 10 days post-PCNL with complaints of recurrent passing of many worm-shaped clots. The patient was admitted and the necessary orders were implemented. The orders included were close observation, complete bed rest, intravenous hydration, check hemoglobin every 6 hours, prepare adequate blood pack cells, and CT-angiography if feasible. His laboratory data upon admission revealed the following levels: $\mathrm{Hb}$ : 8.9, hematocrit: 26.8, BUN: $19, \mathrm{Cr}: 1.25$. The patient consciously refused angiographic embolization with knowledge of the possible complications and deliberately chose repeat nephroscopy.

\section{Endoscopic management}

Patients who did not respond to conservative management prepared for repeat nephroscopy. In our strategies, not responding to conservative management was described:

(1) Intractable clot retention despite bladder irrigation with large bore three-way Foley catheter after 2 days.

(2) Serum hemoglobin decrement despite complete bed rest and blood transfusion after 2 days.

(3) Unstable hemodynamic despite intravenous fluid resuscitation after 24 hours.

(4) Patients who developed recurrent gross hematuria with hemodynamic change after reasonable conservative management.

(5) Present of arterial pseudo aneurysm in imaging reveals during hospitalization.

All repeat nephroscopy were done under spinal anesthesia. With the patient in the lithotomy position, cystourethroscopy was done using a $20 \mathrm{~F}$ cystoscope sheet and a $30^{\circ}$ lens. A $5 \mathrm{~F}$ ureteral catheter was inserted into the involved kidney. The patient was moved into the prone position under observation by the anesthesiologist. Contrast material was injected into the urinary system from the ureteral catheter, and the target calix was accessed (Fig. 1) (the target calix was one that had been dilated in a previous intervention the target calix was full of clot, so it clearly distinguished from other calix.). In some cases, contrast material was extravasated from the previously accessed area, and it was the best guide to the involved calix. Then, a J wire (0.035") was inserted, the tract was dilated with the one-shot dilatation method, and a $30 \mathrm{~F}$ amplatz was inserted. Nephroscopy was performed using a Wolf nephroscope with a $26 \mathrm{~F}$ sheet and $25^{\circ}$ lens. The clot in the target calix was removed with nontooth forceps meticulously; in some cases, pseudo aneurysm were seen and removed (Fig. 2). A council-tipped catheter was inserted into the involved calix, and its balloon was inflated to $4 \mathrm{~mL}$ with water and contrast material (Fig. 3). In our experience, patient

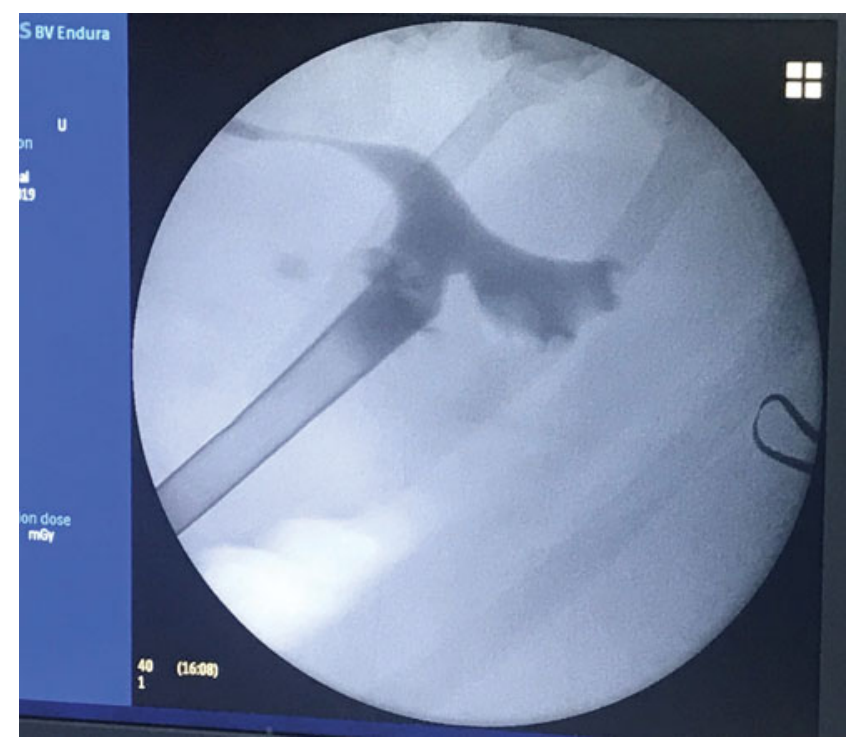

FIG. 1. Contrast material was injected into the urinary system from the ureteral catheter, and the target calix was accessed. 


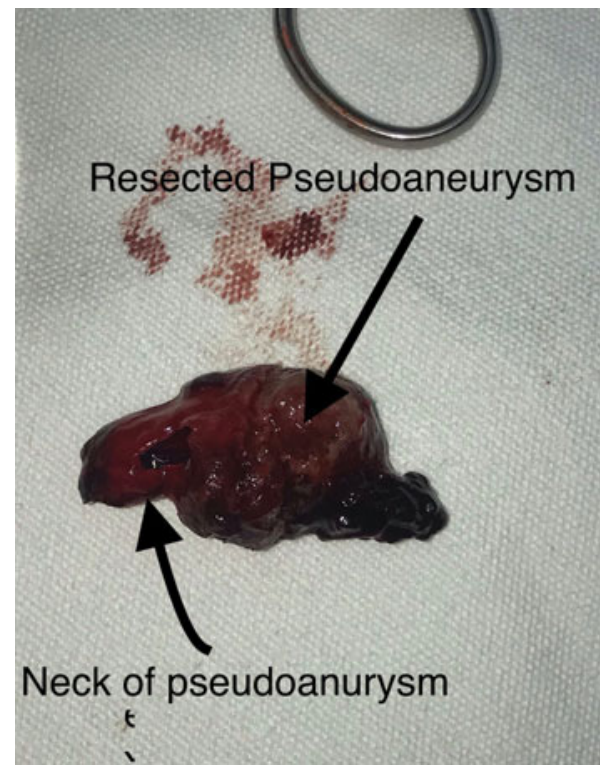

FIG. 2. A pseudoaneurysm that was removed.

could not tolerate the council balloon inflated $>4 \mathrm{~mL}$, and this amount of inflation had become effective and provided acceptable pressure on source of bleeding. The council-tipped catheter was affixed to the skin and clamped (Fig. 4). The color of the urine was clear soon after the operation.

All patients were observed closely for 2 days and then discharged from the hospital with the appropriate antibiotic prescription. Three weeks later, patients returned to have the council-tipped catheter removed. One patient developed a fever of $38^{\circ} \mathrm{C}$ that was relieved by changing the prescribed antibiotic. All patients were further observed for 6 months, and none of them developed any significant complications.

\section{Discussion}

The incidence of delayed life-threatening bleeding, already about $0.3 \%-1.4 \%,{ }^{1}$ is increased by the popularity of

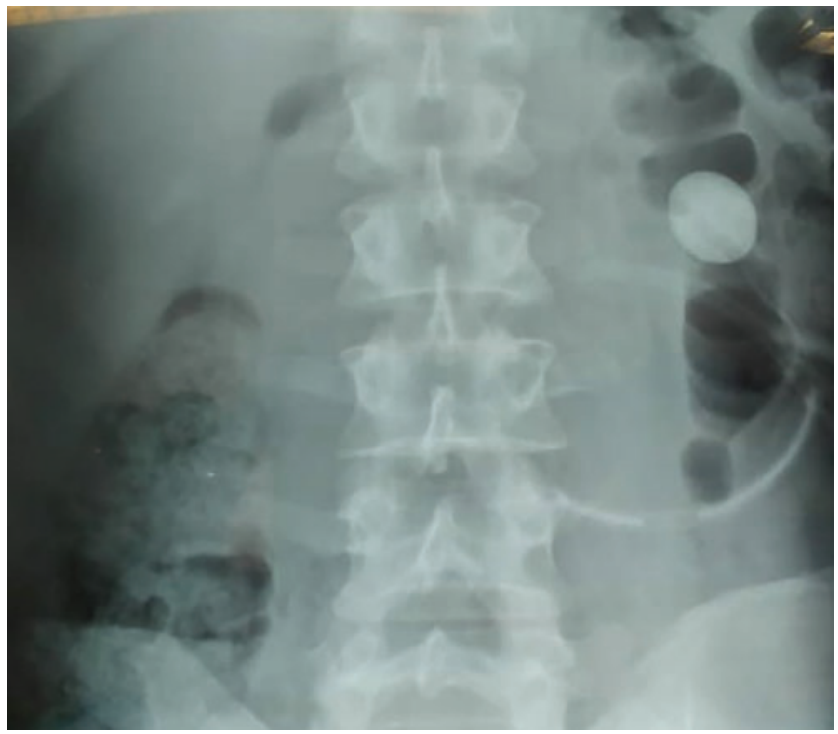

FIG. 3. Balloon was inflated to $4 \mathrm{~mL}$ with water and contrast material.

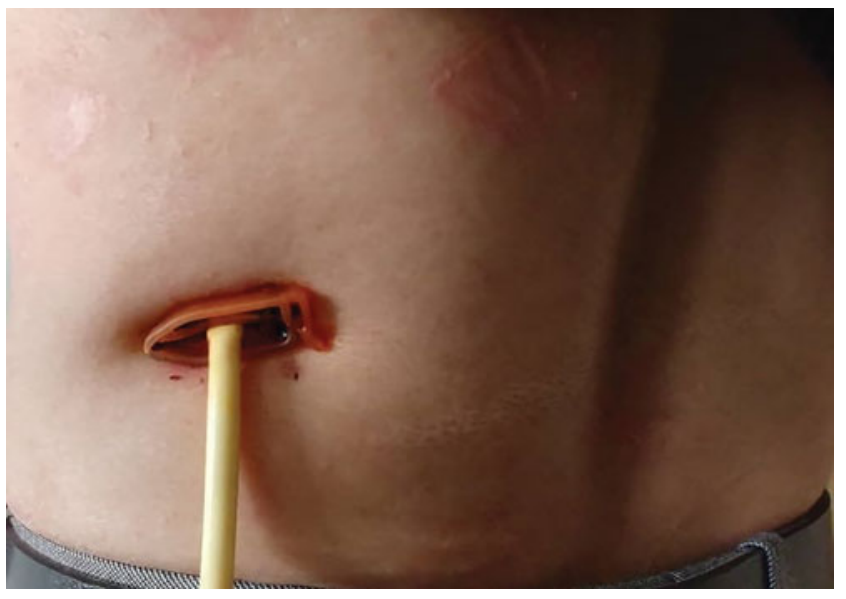

FIG. 4. The council-tipped catheter was affixed to the skin and clamped.

using the percutaneous approach to treat renal stone. ${ }^{1} \mathrm{De}$ layed bleeding is more prevalent in the percutaneous endoscopic resection of the upper urethra than in PCNL. The primary surgical approach to treating renal stone is PCNL. Earlier bleeding in delayed hemorrhage is more closely associated with pseudoaneurysm than with arteriovenous malformation. Large stone burden and renal malformation are the known risk factors of iatrogenic vascular injury. ${ }^{1}$ Although angiographic embolization as the standard treatment has improved over the time and postembolization morbidity has decreased, ${ }^{2}$ there are inevitable complications with angioembolization, most of which are renal insufficiency and embolization of a nontarget area. Retroperitoneal abscess has also been reported but less frequently. ${ }^{2}$ Colonic infarction, skin necrosis, testicular infarction, and coil migration have also been reported. ${ }^{3}$ In some cases, a missed diagnosis of a tiny pseudoaneurysm has led to ineffective angiographic embolization. ${ }^{4}$ Recurrent bleeding that is not a response to standard angioembolization may require reembolization, partial nephrectomy, or total nephrectomy. ${ }^{2}$ The leading outcome of this failure is nephron loss, which may be disastrous in patients with chronic renal failure or a single kidney. Patients with chronic renal failure are prone to develop acute tubular necrosis associated with the contrast material. $^{2}$

The balloon tamponade with kaye nephrostomy is well known as treatment of intraoperation bleeding during PCNL that does not respond to simple nephrostomy insertion. Using council catheter for management of delayed hemorrhage after PCNL is a novel approach with acceptable efficacy.

Our experience opens a new window in the management of post-PCNL delayed bleeding without significant complications. Nephron sparing and avoidance of nephrectomy are the main advantages of this approach. Its difficulties lie in the complexity of the procedure and will be solved by an expert endourologist. We believed in the superiority of the advantages over the difficulties of this surgical approach.

\section{Conclusion}

To the best of the authors' knowledge, the approach discussed herein is an ideal technique for cases in which 
angioembolization is not feasible, and it may play an important role in the management of angioembolization failure.

\section{Disclosure Statement}

No competing financial interests exist.

\section{Funding Information}

No funding was received for this article.

\section{References}

1. Un S, Cakir V, Kara C, et al. Risk factors for hemorrhage requiring embolization after percutaneous nephrolithotomy. Can Urol Assoc J 2015;9:E594-E598.

2. Palmerola R, Patel V, Hartman C, et al. Renal functional outcomes are not adversely affected by selective angioembolization following percutaneous nephrolithotomy. Asian J Urol 2017;4:27-30.

3. Giusti G, Lisa A. Massive migration of embolization coils inside the renal pelvis. A rare complication that can be approached through percutaneous surgery. Cent Eur J Urol 2018;71:467-469.

4. Ren YM, Wu XM, Wen Y, et al. [Recurrent bleeding following the renal artery embolization treating post-percutaneous nephrolithotomy hemorrhage: Causes and countermeasure]. Zhonghua yi xue za zhi 2017;97:22-25.
Address correspondence to: Arsalan Aslani, MD Urology and Nephrology Research Center Shahid Labbafinejad Medical Center Shahid Beheshti University of Medical Sciences 9th Boostan Street, Pasdaran Avenue Tehran 1666663111 Iran

E-mail: aslani.arsalan@gmail.com

$\begin{aligned} & \text { Abbreviations Used } \\ \mathrm{BUN} & =\text { blood urea nitrogen } \\ \mathrm{Cr} & =\text { creatinine } \\ \mathrm{CT} & =\text { computed tomography } \\ \mathrm{Hb} & =\text { hemoglobin } \\ \mathrm{PCNL} & =\text { percutaneous nephrolithotomy }\end{aligned}$

Cite this article as: Nouralizadeh A, Aslani A, Ghanaat I, Bonakdar Hashemi M (2020) Percutaneous endoscopic treatment of complicated delayed bleeding postpercutaneous nephrolithotomy: A novel suggestion, Journal of Endourology Case Reports 6:3, 124-127, DOI: $10.1089 /$ cren.2019.0091. 